Handlungsmöglichkeiten für eine nationale Politik der Nachhaltigkeit

\section{Nachhaltigkeit und Globalisierung}

\section{Im Zuge der Globalisierung seien nationale Standorte weitgehend austauschbar und aufgrund des zunehmenden Standortwettbewerbs seien nationale Hand- lungsspielräume entsprechend gering - so wird häufig argumentiert. Gängige Meinung isł auch, daß Globalisierung zu grundsätzlich negativen ökologischen Effekten führe. Es gilt, diese Vorstellungen zu relativieren und Handlungsmög- lichkeiten für eine Politik der Nachhaltigkeit aufzeigen.}

\section{Von Ulrich Petschow und Jürgen Meyerboff} lobalisierung wird in der Regel mit umfassender Entgrenzung, Nachhaltigkeit mit Begrenzung gleichgesetzt. Entgrenzung und Begrenzung sind freilich nur auf den ersten Blick Gegensätze. Durch die beschleunigte Globalisierung ökonomischer Aktivitäten werden die Grenzen des Planeten Erde auf eine neue Weise berührt. Die dichter werdenden ökonomischen Vernetzungen holen insoweit nur nach und verschärfen zugleich, was hinsichtlich der Umweltwirkungen von wirtschaftlichen Operationen bereits Realität ist. In beiden Fällen werden nationalstaatlich-territoriale Grenzen überwunden und bilden sich globale Zusammenhänge heraus. So gesehen sind Globalisierung und Nachhaltigkeit miteinander verbunden und verweisen auf die sich daraus ergebenden Handlungschancen und -risiken.

\section{Auswirkungen auf die Umwelt}

Die Auswirkungen der Globalisierung auf die Umwelt hängen von einer Vielzahl von Faktoren in den jeweiligen Ländern $a b$, so z.B. dem Entwicklungsstadium, den Machtverhältnissen und damit dem Grad der Marktöffnung, den Wachstumsbedingungen vor Ort und der Stellung im internationalen System. Allgemeine Aussagen sind daher nur begrenzt möglich. Zudem ist festzuhalten, daß sich auch die Ausformung des „traditionellen wirtschaftlichen Fortschritts- und Wachstumsmodells" in den Industriestaaten selbst stark unterscheidet. (Beispiel Energieverbrauch USA - Europa). Doch läßt sich grob zwischen zwei Auffassungen von Globalisierung unterscheiden:

- Zum einen kann Globalisierung als die weltweite Durchsetzung eines wirtschaftlichen Entwicklungsmodells verstanden werden. Unabhängig von der Öffnung der Volkswirt- schaften wird die Vorstellung von Entwicklung als industrieller Entwicklung und die damit verbundenen Konsummuster global übernommen.

- Zum anderen kann Globalisierung aber auch als ein umfassender Prozeß der Liberalisierung, der Einfiihrung marktwirtschaftlicher Verhältnisse, des Abbaus von Handelshemmnissen und letztlich der Schaffung eines globalen Marktes verstanden werden.

Beide ,Typen“ sind auseinander zu halten, wenn die Auswirkungen auf die ökologische Nachhaltigkeit beurteilt werden sollen. Beim ersten Typ wird der Schwerpunkt auf eine vom Weltmarkt abgekoppelte industrielle Entwicklung gelegt. So haben die ehemaligen sozialistischen Länder, die lange Zeit als Repräsentanten dieses Typs galten, einen besonders ressourcenintensiven Entwicklungspfad beschritten, der weder im ökologischen noch im ökonomischen Sinne nachhaltig war. Dies gilt auch für einige Entwicklungsländer, die ihre wirtschaftliche Entwicklung durch den Schutz ihrer Industrie mit Hilfe von Marktabschottungen fördern wollten. Beim zweiten Typ wird Globalisierung verstanden als Liberalisierung des Welthandels und der globalen Geld- und Finanzmärkte und kann bezogen auf die ökologische Nachhaltigkeit ambivalente Wirkungen haben. Infolge der Liberalisierung kann es zu einer höheren Ressourceneffizienz und dem Austausch nachhaltiger Technologien kommen, aber auch zur Infragestellung existierender (Umwelt-) Standards. Außerdem kann das aufgrund der Globalisierung zu erwartende Wirtschaftswachstum zu einer erheblichen Gefährdung der natürlichen Umwelt führen (Scale-Effekt). Die Diskussion um den Zusammenhang von Wirtschaftswachstum und Umweltbelastung wird weiterhin sehr kontrovers geführt. So zeigen empirische Untersuchungen oft einen glockenförmigen Verlauf des Zusammenhangs zwischen Umweltqualität und Wirtschaftswachstum (1). Dahinter steht die Annahme, daß vor allem zu Beginn von Wachstumsprozessen eine enge - überproportionale - Koppelung zwischen den beiden Größen besteht, im Verlaufe des Wachstumsprozesses es aber zu einer Entkoppelung von Umweltbelastung und Wachstum komme.

Diese Auffassung wird allerdings in Frage gestellt. (So verweisen Arrow et al. (2) darauf, daß dieser Zusammenhang mit Vorsicht zu interpretieren ist.) Insbesondere für Umweltbelastungen, die durch Akkumulation entstehen, also gekennzeichnet sind durch time-lags und räumliche Differenzierung der anfallenden Kosten (Beispiel Treibhauseffekt), wurde dieser Zusammenhang nicht nachgewiesen. Weiterhin ist diese Annahme problematisch, wenn bei bestimmten Ressourcen Rückkoppelungseffekte auftreten oder wenn die Reduktion der Umweltbelastung an einer Stelle mit einer Zunahme an andere Stelle verbunden sein kann. Sowohl die Globalisierungs- als auch die Wachstumsfolgen erfordern daher eine differenzierte Betrachtung.

\section{Triadisierung und zunehmender Wettbewerbsdruck}

Wie Kurt Hübner (in diesem Heft S. 11) feststellt, handelt es sich bei der sogenannten Globalisierung wesentlich um eine Triadisierung, das heißt um die Bildung von drei Wirtschaftsblöcken. Innerhalb dieser Blöcke stehen die nationalen Räume wiederum miteinander in Konkurrenz. Gleichzeitig bilden sich spezifische Formen der Arbeitsteilung aus, die die Wettbewerbsfähigkeit der Blöcke in der globalen Ökonomie stärken. Die Folge ist eine Verwischung von Konturen nationaler Märkte und Produktionsstandorte. Das Projekt der (Block-) Integration ist allerdings in seinem Umfang begrenzt. Insbesondere die Nicht-Herstellung eines einheitlichen europäischen Sozial-, Steuer- und Umweltraumes bewirkt eine Verengung der politischen Gestaltungspotentiale, da in diesen Politikarenen nach wie vor nationale Regelungen den Vorrang haben. Besonders deutlich wird dies in der Steuergesetzgebung. Hier werden den Unternehmen neue Bündel an Ausweichmöglichkeiten angeboten und ein internationaler Wettlauf um die Senkung von Steuern und auch Umweltstandards in Gang gesetzt, der als ein ,race to the bottom" bezeichnet werden kann, das langfristig zerstörerische Folgen für die nationalen Räume zeitigen kann. 
Die zunehmende Konkurrenz auf den globalisierten Märkten spiegelt sich in der Diskussion um den Standort Deutschland wider. Demnach werden zwei Typen von ,Krisen“ unterschieden (3):

- Die Interpretation der Standortkrise als Kostenkrise, deren Ursachen in der Höhe der Arbeitskosten, den Steuern und Abgaben und der Regulierung, insbesondere auch im Umweltbereich, liegen (Therapievorschlag entsprechend Reduktion der Faktorkosten).

- Die Krise als Ergebnis einer mangelnden Produktivitäts- und Innovationsdynamik innerhalb des privaten und öffentlichen Sektors in Deutschland (Therapievorschlag: Innovation und Qualitätsdifferenzierung).

Folgt man der zweiten Auffassung, dann überwiegt in Deutschland eine diversifizierte Qualitätsproduktion, deren Grundlage qualifizierte Arbeitskräfte und die von Ingenieuren und Management entwickelten technisch-orientierten Problemlösungsstrategien bilden; verbunden damit ist eine starke Produzenten-, aber nur eine schwach entwickelte Kundenorientierung in den Prozeßketten. Zentraler Schwachpunkt ist der Mangel an Kommunikation und Kooperation sowohl an den Schnittstellen der einzelnen Unternehmensbereiche und zum Markt hin. Gerade diese Mängel haben eine zunehmende Bedeutung für die Einschätzung der Wettbewerbsfähigkeit.

Wie Hartmut Hirsch-Kreinsen (in diesem Heft S.17) zeigt, verändern sich die Unternehmensstrukturen zur Zeit umfassend. Handlungsoptionen entstehen durch die verringerte Bedeutung des Absatzes von (Massen-) Produkten und der verstärkten Bedeutung von angepaßten bzw. maßgeschneiderte Lösungen. Damit ist auch eine grundsätzliche Blickwende von der Inputzur Outputorientierung verbunden, künftige Marktchancen und Marktpotentiale rücken in den Vordergrund. Zudem nimmt die Orientierung an den sogenannten ,Lead Markets“ (Frieder Meyer-Krahmer ebenfalls in diesem Heft S. 20) zu, da dort neue Trends gesetzt bzw. wahrgenommen werden können. Die von Porter und anderen Autoren festgestellte, und auch von der Innovationsforschung bestätigte, veränderte Ausrichtung der Unternehmen wird auch für Deutschland zunehmend wichtig. Entscheidend für den Standort Deutschland sind von daher Innovationen und deren Umsetzung zur Überwindung der benannten Produktivitäts- und Innovationskrise. Damit können Nachhaltigkeitsanforderungen - als Modernisierungsanforderungen - eine wesentliche Rolle auch für den Standort spielen.

\section{Austauschbarkeit der Standorte?}

Auf den globalen Märkten zeigen sich gegenwärtig gegenläufige Entwicklungen. So ist einerseits ein umfassender Trend der Homogenisierung der globalen Nachfrage zu beobachten. Er geht einher mit der umfassenden Möglichkeit zur Globalisierung der Produktion. Andererseits findet jedoch auch eine Fragmentierung der Märkte statt: Eine spezialisierte Nachfrage führt zur Marktdifferenzierung und zur Anpassung der Produzenten an diese Nachfrage. Spezifischen Marktkenntnissen und der Nähe zum Markt kommt eine zunehmende Bedeutung zu. Dadurch können die Spezialisierungsvorteile, die bestimmte Orte und Regionen haben, relevanter werden und sich dadurch Handlungsspielräume eröffnen

Diese Tendenzen spiegeln sich auch im Spannungsverhältnis von Territorialisierung und Globalisierung der Produktionssysteme wider: territorial gebundene Systeme können in den internationalen Austausch einbezogen werden, ohne daß ihr Territoriumsbezug sich zwangsläufig löst. Sie sind territorial und global zugleich. Dies gilt für High-Tech Regionen wie Silicon Valley oder Industriedistrikte z.B. in Italien. Die Vorteile ergeben sich nicht aufgrund der Isolation der Regionen, sondern aufgrund der ,ongoing reinvention of relational assets in the context of high levels of geographical openness in trade and communications" (4).

Damit kommt den Standortbedingungen in den unterschiedlichen Staaten und Regionen eine wachsende Bedeutung für die Wettbewerbsfähigkeit der ansässigen Unternehmen zu. Neue theoretische und empirische Untersuchungen betonen vor allem die Bedeutung von Netzwerkstrukturen und die Herausbildung von dynamischen Vorteilen (Humankapitalbildung, Informations- und Wissenstransfer). Staatliche Maßnahmen, auch die Umsetzung der Nachhaltigkeitsanforderungen, können vielmehr die Rahmenbedingungen für die dynamische Wettbewerbsfähigkeit der Unternehmen stärken. (Porter Hypothese).

\section{Die Dynamik der Globalisierung}

Das im folgenden dargestellte Konzept der Territorialisierung von Storper (4) versucht, diejeni-

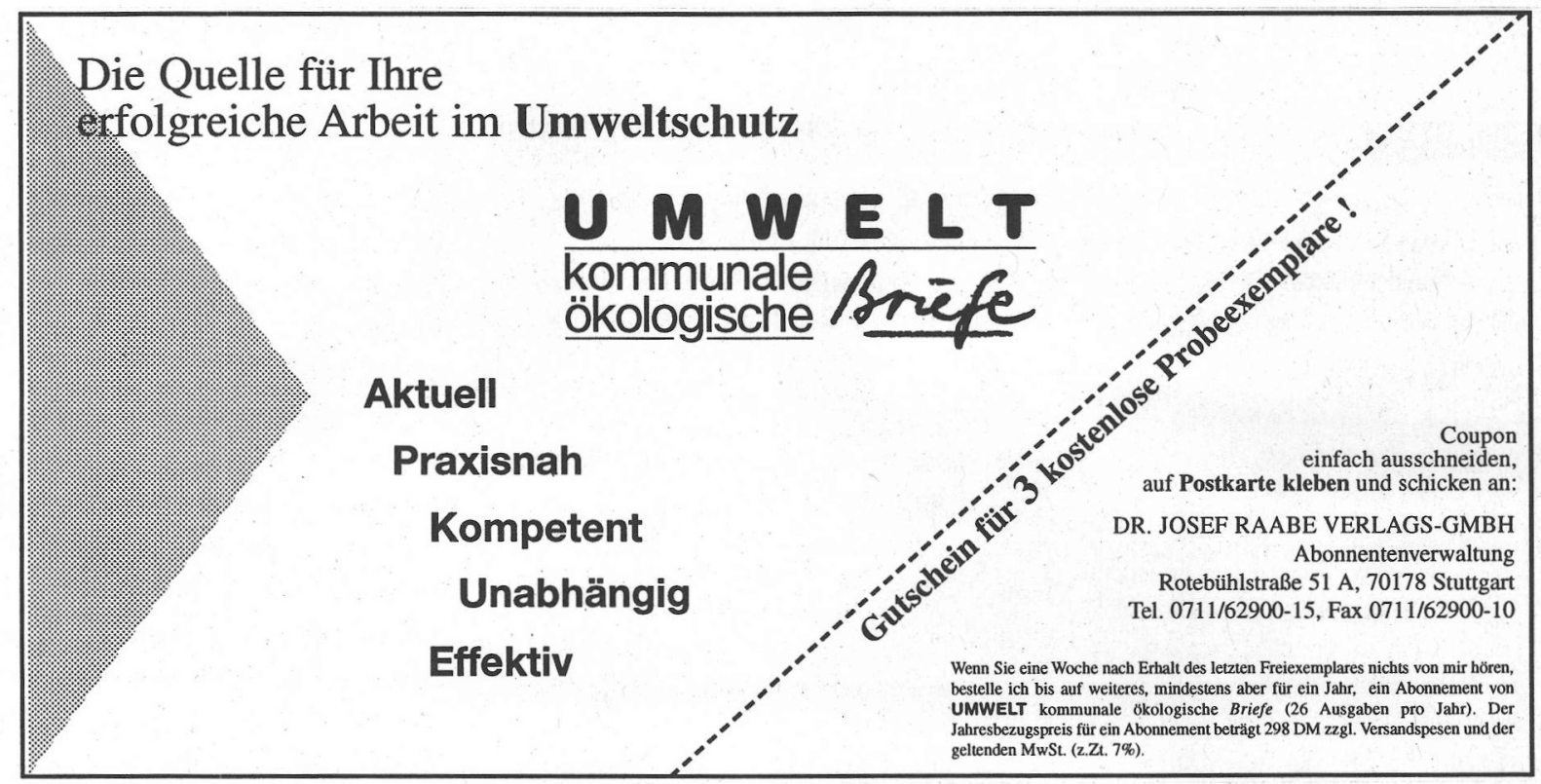


gen Faktoren zu identifizieren, die geeignet sind, die territoriale Gebundenheit von Produktionen darzustellen. Er unterscheidet dazu idealtypisch: - Erstens die territorial völlig ungebundene Ökonomie (economy of flows and substitutions): Die Produktion ist an allen Orten der Welt grundsätzlich möglich. Eine Anbindung an bestimmte Territorien existiert nicht. In Abhängigkeit von den ,sunk costs“ kann die Produktion jederzeit an ,irgendeinen“ Ort verlagert werden. Standortverlagerungen stellen für die Unternehmen insofern kein Problem dar. Diese „Flow Ökonomie“ kann die Konsequenz der Verbesserung der Transportmöglichkeiten oder auch der Homogenisierung der lokalen und regionalen Besonderheiten (z.B. Konsumstrukturen) sein.

- Zweitens die territorial völlig gebundene Ökonomie (economy of interdependencies and specifities):

In diesem Fall sind die wirtschaftlichen Aktivitäten von den Ressourcen des Territoriums abhängig, z.B. wenn territorial gebundene Technologie- und Arbeitsinputs existieren, die überhaupt keine Substitute haben. Dabei ist insbesondere die Qualifikation der Beschäftigten (Kreativität) von Bedeutung, oder die Existenz von speziellen Konventionen bzw. Know-how. Das Angebot von derartigen Qualifikationsprofilen in einem Nationalstaat oder einer Region ist zumindest mittelfristig unelastisch. Bedeutend ist vor allem, daß regionale und nationale Produktionssysteme auf die Interdependenz zwischen Organisationen und Personen abstellen, die eine spezifische Ausgestaltung der Vorteile beinhalten. Die Realität bewegt sich, je nach Branche und Unternehmen, zwischen den beiden Extrempunkten. Storper entwickelte daher eine Matrix, die als Bezugspunkt die territoriale (Un-) Gebundenheit der Produktion hat. Wenn also die Globalisierung gegenüber der territorial gebundenen ökonomischen Organisation gewinnt, dann muß sich dies in einem abnehmenden Grad der Territorialisierung äußern. In der Grafik ist diese in Feldern dargestellt.

Feld eins stellt Produktionen dar, die umfassend territorialisiert und zugleich hochgradig internationalisiert sind. Es handelt sich somit um Produktionen, die von nicht substituierbaren regionalen Faktoren abhängig sind, die aber transferiert werden können.

In Feld zwei sind die Fälle einbezogen, deren territoriale Gebundenheit gering und deren Produktion hochgradig flexibel ist (standardisierte Massenprodukte). Die entsprechenden Produktionsstandorte sind in starkem Maße substituierbar (typischer Fall der Globalisierung).

In Feld drei befinden sich stark territorialisierte Systeme mit geringen internationalen Verbindungen, diese sind aufgrund nicht substituierbarer lokaler Vorteile und spezieller Nachfrage territorial gebunden.

Feld vier beinhaltet Bereiche mit geringer territorialer Gebundenheit und geringen Niveaus der internationalen flows der Warenströme und Produktionssysteme, wie beispielsweise der lokale Handel und lokale Dienstleistungen.

Interessant sind vor allem die Übergangsprozesse zwischen den unterschiedlichen Feldern: Einen typischen Fall von Globalisierung stellt der Wandel von Feld drei zu Feld zwei dar, wenn z.B. aufgrund sinkender Transportkosten der Marktzugang

\section{Die Dynamik der Globalisierung}

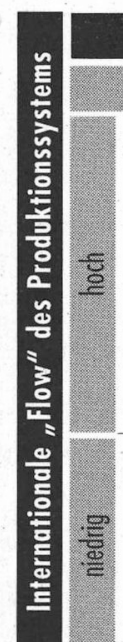

Territorialisierung des Produktionssystems

hoch

Feld 1: hohe Territorialisierung und internationaler Handel

a) Interner Unternehmenshandel:

-Zwischenprodukte von FDI (foreign direct investment)

- territoriale Kerne beliefern internationale Mörkte

b) industrielle Distrikte

c) Handel zwischen Unternehmen

Feld 3: hohe Territorialisierung geringer internationaler Austausch

Produktionen, die auf die Region ausgerichtet sind: spezialisierte Nachfrage, geringe internationale Konkurrenz.

Quelle: Storper (1995) erleichtert wird. Was die Frage der Handlungsmöglichkeiten unter den Bedingungen der Globalisierung angeht, so sind gerade die Wechsel von den Feldern drei und vier zu Feld eins untersuchendwert. Diese Wechsel bedeuten, daß ehemals hochgradig territorial gebundene Systeme internationalisiert werden, indem sie nun ihre Produkte weltweit vermarkten, ohne aber dabei ihre ortsgebundenen Bedingungen der Produktion zu verlieren. Desweiteren ist die Transformation der Produktionssysteme von Feld zwei in solche von Feld eins von Bedeutung. Hierbei werden hochgradig internationalisierte, aber nicht lokal gebundene Systeme zunehmend territorialisiert. Dies ist dort der Fall, wo es zu einer Differenzierung eines Massenproduktes kommt und sich die Möglichkeit eröffnet, daß neue standortspezifische relative Vorteile für die Produktion entstehen.Insofern ist hervorzuheben, daß Globalisierung trotz umfassender Veränderungen der Verkehrs- und Kommunikationstechnologien nicht zu einer durchgängigen Homogenisierung führt. Sie ist vielmehr zugleich durch die Segmentierung bzw. Fragmentierung einzelner Räume und Märkte gekennzeichnet. Dieses wahrzunehmen eröffnet wiederum Handlungsspielräume auch für eine nationale Politik der Nachhaltigkeit.

\section{- Handlungsspielräume}

Die Globalisierungsprozesse stellen eine erhebliche Herausforderung an die Nationalsstaaten dar. Doch läßt sich daraus nicht schließen, daß die Anforderungen der Globalisierung per se nicht mit denen einer Politik der Nachhaltigkeit zu vereinbaren sind.

1. Wie Frieder Meyer-Krahmer aufzeigt, kann die Umsetzung von Anforderungen der ökologischen Nachhaltigkeit eine wesentliche Rolle für die Attraktivität eines Standortes für die Forschung- und Entwicklung-(FuE)-Kompetenzzentren multinationaler Unternehmen darstellen. Die Thesen Porters zur Bedeutung nationaler Bedingungen zur Ausprägung der Wettbewerbsfähigkeit von Unternehmen finden im Bereich von FuE ihre Bestätigung. Eine umfassende Politik der ökologischen Nachhaltigkeit, kann insofern eine Basis darstellen für die Entwicklung der ökonomischen und sozialen Nachhaltigkeit. 2. Der Zugriff des Weltmarktes auf die nationalen Märkte muß zudem nicht zwangsläufig und umfassend ökologisch kontraproduktive Effekte haben, sondern kann vielmehr geeignet sein, „Handlungsblockaden“ für die Politik wie ökologisch kontraproduktive Monopolstellungen in 
den jeweiligen Ländern aufzuweichen. So fïhrt im Bereich der Landwirtschaft die Begrenzung der Protektionsmöglichkeiten aufgrund internationaler Abkommen wie dem GATT (General Agreement on Tarrifs and Trade) zu der Möglichkeit, Schritte in Richtung Nachhaltigkeit einzuschlagen: ökologische Leistungen können honoriert werden, ökologisch kontraproduktive Subventionen müssen aber abgebaut werden. Ähnliches gilt für die Deregulierung der Elektrizitätsmärkte, die allerdings darüber hinaus einer Re-Regulierung bedürfen, um Wettbewerb dauerhaft zu ermöglichen und ökologisch nachteilige Entwicklungen zu vermeiden.

3. Doch gibt es auch etliche Handlungsspielräume, die nicht mit der Globalisierung in Verbindung zu bringen sind. Zum Teil handelt es sich dabei um Probleme, die schon seit langem in der Umwelt- bzw. Naturschutzpolitik diskutiert wurden. So ist der Bodenschutz und insbesondere die Frage der Versiegelung nur begrenzt mit der Globalisierung in Verbindung zu bringen. Wesentliche Triebkräfte sind vorrangig national wie Lebensstil und Konsumverhalten. Gerade der Bereich der Konsumstrukturen bleibt grundsätzlich weitgehend den Eingriffsmöglichkeiten einer nationalen Politik der Nachhaltigkeit unterworfen.

\section{Anmerkung}

Die Ausführungen gehen auf ein Gutachten für die EnqueteKommission "Schutz des Menschen und der Umwelt" unter dem Titel Auswirkungen der Triebkräfte und Trends der Globalisiserung auf eine Politik der Nachhaltigkeit" zurück, das vom löW erstellt wurde. Eine Veröffentlichung ist für März/April 1997 von der Enquete-Kommission geplant. Literatur

1) G.M. Grossman, A.B. Krueger: Economic Growth and the Environment. In: Quarterly Journal of Economics, 1995, 353-377

2) K. Arrow: Economic Growth, Carrying Capacitiy, and the Environment. In: Ecological Economics, 1995, Vol. 15, 91 . 95.

3) F. Naschold: Jenseits des baden-württembergischen "Rxceptionalims": Strukturprobleme der deutschen Industrie. Stuttgart 1994

4) M. Storper: Territories, flows and hierarchies in the global economy. In: Außenwirtschaft 50, 1995, 11

\section{Die Autoren}

Ulrich Petschow und Jürgen Meyerhoff sind wissenschafliche Mitarbeiter des löW im Forschungsfeld "Umwelipolitik und-ökonomie" Kontakt: IÖW Berlin, (030) 884594.0
Globalisierung der Industrie und Wandel von Unternehmensstrategien

\section{Netzwerkinterne Konkurrenz lokaler Unternehmenseinheiten}

\section{In Reaktion auf die vielfältigen Strukturveränderungen des Weltmarktes erge- ben sich beträchtliche Strategie- und Organisationsveränderungen bei Indu- strieunternehmen. Transnationale Netzwerke werden aufgebaut, die sowohl der Koordination dienen als auch eine Konkurrenzsituation einzelner Unterneh- menseinheiten schaffen. Gerade diese netzwerkinterne Konkurrenz könnte die Nutzung von Umweltstandards zur Förderung von Absatzchancen bewirken.}

D

Von Hartmut Hirsch-Kreinsen as Schlagwort Globalisierung beherrscht seit längerem die gesellschaftspolitische Debatte - nicht nur in Deutschland, sondern auch in anderen Ländern. Realer Kern dieser Debatte sind die massiv ansteigenden weltwirtschaftlichen Verflechtungen der letzten zehn bis 15 Jahre. Sie umfassen den kontinuierlich steigenden Welthandel, die weltweite Vernetzung der Finanz- und Kapitalmärkte sowie die sich immer weiter ausdehnenden Aktivitäten internationaler Unternehmen. Einen deutlichen Hinweis auf die wachsende Bedeutung internationaler Unternehmen gibt der schnelle Anstieg der ausländischen Direktinvestitionen seit Mitte der 80er Jahre. Dies gilt generell für alle westlichen Industrieländer, insbesondere aber für die Bundesrepublik Deutschland. Ausgehend von einem im internationalen Vergleich relativ niedrigen Vermögensbestand hat sich der Gesamtbestand ausländischer Direktinvestitionen deutscher Unternehmen nach Angaben der Bundesbank zwischen 1985 und 1994 von rund 150 auf knapp 350 Milliarden Mark mehr als verdoppelt. 1995 stiegen die Auslandsinvestitionen nochmals geradezu sprunghaft an und Deutschland steht nach Japan und den USA an dritter Stelle aller im Ausland investierenden Länder. Greift man den industriellen Sektor heraus, so zeigt sich ein nicht ganz so steiler, freilich auch signifikanter Anstieg der Direktinvestitionen: im Zehnjahreszeitraum von 1985 bis 1994 stieg der Bestand der Direktinvestitionen des verarbeitenden Gewerbes im Ausland von rund 89 auf 165 Milliarden Mark (1).

Damit verbinden sich, wie im folgenden genauer gezeigt werden soll, beträchtliche Strategieund Organisationsveränderungen von Industrie- unternehmen. Fraglos verändern sich damit auch die Bedingungen für die Durchsetzung umweltpolitischer Ziele.

\section{Transnationale Strategien}

Sowohl Ursache als auch Folge der fortschreitenden Internationalisierung der industriellen Produktion sind Strukturveränderungen des Weltmarktes. Angesichts einer stagnierenden oder rückläufigen Nachfrage im Inland, einer wachsenden Konkurrenz ausländischer Unternehmen vor allem auch aus neuindustrialisierten Ländern und der währungs- und handelspolitischen Turbulenzen auf dem Weltmarkt drängen die Unternehmen verstärkt ins Ausland. Beobachtbar ist ein neuer Typus von Unternehmensstrategie, der als "transnationale“ Strategie bezeichnet werden kann (2). Mit dieser Strategie verfolgen die Unternehmen drei teilweise einander widersprechende Hauptziele:

- Durch eine weltweite Standardisierung und Massenproduktion bestimmter Produkte und Produktkomponenten sollen Produktivitätsvorteile erreicht werden, um der intensiven Konkurrenz begegnen zu können.

- Stagnierender Absatz und risikoreiche Währungsturbulenzen sollen durch eine flexible und an lokale Marktbedingungen angepaßte Produktion bewältigt werden und durch die damit gewonnene räumliche Nähe zu den Abnehmern der Produkte soll neue Absatzchancen eröffnen.

- Und nicht zuletzt geht es um die Abstimmung, die Rationalisierung und die Beschleunigung der Innovationsprozesse in den weltweit verteilten Produktionsstandorten. Einschlägiges Stichwort ist hier die permanente Verkürzung der „time to market“. 
(c) 20I0 Authors; licensee IÖW and oekom verlag. This is an article distributed under the terms of the Creative Commons Attribution Non-Commercial No Derivates License (http://creativecommons.org/licenses/by-nc-nd/3.o/), which permits unrestricted use, distribution, and reproduction in any medium, provided the original work is properly cited. 